\title{
Circle of Willis variations and artery diameter measurements in the Turkish population
}

\author{
I.Ö. Yeniçeri ${ }^{1}$, N. Çullu ${ }^{1}$, M. Deveer ${ }^{1}$, E.N. Yeniçeri ${ }^{2}$ \\ ${ }^{1}$ Department of Radiology, Muğla Sitkı Koçman University, Faculty of Medicine, Muğla, Turkey \\ ${ }^{2}$ Department of Family Medicine, Muğla Sıtkı Koçman University, Faculty of Medicine, Muğla, Turkey
}

[Received: 24 September 2016; Accepted: 18 December 2016]

Background: The aim of this study was to evaluate the frequency of circle of Willis (COW) variations and the measurements of the COW vessel diameters in the Turkish population.

Materials and methods: The patient population for this single-centre, retrospective study was formed of patients who had brain magnetic resonance angiography (MRA). A total of 384 subjects were included in the study. The three-dimensional time-of-flight (TOF) technique was used for MRA imaging to evaluate the anatomy of the COW. Variations in the COW were recorded. The diameters of the critical arteries of the COW were measured.

Results: Adult configuration of the COW was determined in 326 (85\%) cases, foetal configuration in $50(13 \%)$ cases and transitional configuration in $8(2 \%)$ cases. The COW was normal in 328 (85.4\%). In the remaining 56 (14.6\%) cases, agenesis was determined in 62 vessels. The mean calibrations of the vessels were measured as $2.85 \mathrm{~mm}$ in the basilar artery, $4.24 \mathrm{~mm}$ in the right internal carotid artery (ICA), $4.32 \mathrm{~mm}$ in the left ICA, $1.58 \mathrm{~mm}$ in the right $A 1,1.64 \mathrm{~mm}$ in the left $A 1,2.13 \mathrm{~mm}$ in the right $M 1,2.10 \mathrm{~mm}$ in the left $M 1,1.80 \mathrm{~mm}$ in the right $P 1,1.88 \mathrm{~mm}$ in the left P1, $1.12 \mathrm{~mm}$ in the right posterior communicating artery, and $1.12 \mathrm{~mm}$ in the left posterior communicating artery.

Conclusions: Circle of Willis variations may show geographic and ethnic differences. Knowledge of the frequency and types of variation in the population is important for neurosurgeons and in radiological interventional procedures as a guide for entry and in respect of collateral which could develop later. (Folia Morphol 2017; 76, 3: 420-425)

Key words: circle of Willis, arterial diameter, variation, magnetic resonance angiography

\section{INTRODUCTION}

Circle of Willis (COW) is of critical importance in brain supply. The morphology of COW is not constant and may have many variations [9]. A normal COW can compensate for the absence of one of the four feeding arteries [10]. Knowledge of these variations is important for brain surgeons considering intervention to the intracranial arteries and for interventional radiologists [4].
With developments in rapid imaging techniques, the COW, which has been traditionally evaluated with angiography, can now be evaluated with magnetic resonance angiography (MRA) and computed tomography angiography (CTA). The clinical use of these two techniques has increased as they are relatively non-invasive methods compared to angiography. High contrast is provided between stationary tissues 
and blood flow on technologically advanced three-dimensional (3D) time-of-flight (TOF) MRA [6].

Circle of Willis variations have been examined in various populations and age groups $[1,5,7]$. However, the age groups in the study populations were extremely large; they also included the elderly. There have been very few studies conducted on a young population. The aim of this study was to evaluate the frequency of COW variations and the measurements of the COW vessel diameters in the Turkish population.

\section{MATERIALS AND METHODS}

\section{Study population}

Ethical approval for the study was given by the Ethics Committee of Muğla Sıtkı Koçman University. The patient population for this single-centre, retrospective study was formed of patients who had brain MRA at the Radiology Clinic with complaints of headache. A total of 424 Caucasian subjects who met the study criteria between December 2012 and February 2016 were included in the study. As the magnetic resonance images (MRI) were not diagnostic in 40 cases, they were excluded from the study. A retrospective evaluation was made of the MRI of a total of 384 patients, comprising 150 males (18-40 years, mean age 30.84 year) and 234 females (18-40 years, mean 29.14 year) and diameters were measured. Mean age of the study participants were 29.78 years (range, 18-40 years). People over 40 years old were excluded because they may have atherosclerosis-related, misleading hypoplasia/aplasia. Those with poor quality images, previous surgery, aneurysm, arteriovenous malformation or tumour previously known or diagnosed during tests, those with a history of stroke and those undergoing therapeutic radiation were also excluded.

\section{Imaging and evaluation}

Magnetic resonance imaging examinations were made using 1.5 T MR scanner (Siemens Essenza with TIM+DOT, Erlangen, Germany). The 3D TOF technique was used for MRA imaging with the parameters of slice thickness $0.65 \mathrm{~mm}$, TR $28 \mathrm{msn}$, TE $7.15 \mathrm{msn}$, flip angle $25^{\circ}$, NEX 1, FOV $180 \mathrm{~cm}$, matrix $256 \times 241$. Evaluation was made from the images of source axial dataset and maximal intensity project (MIP).

The MRA images were evaluated by a radiologist with 18 years of experience. To increase the sensitivity
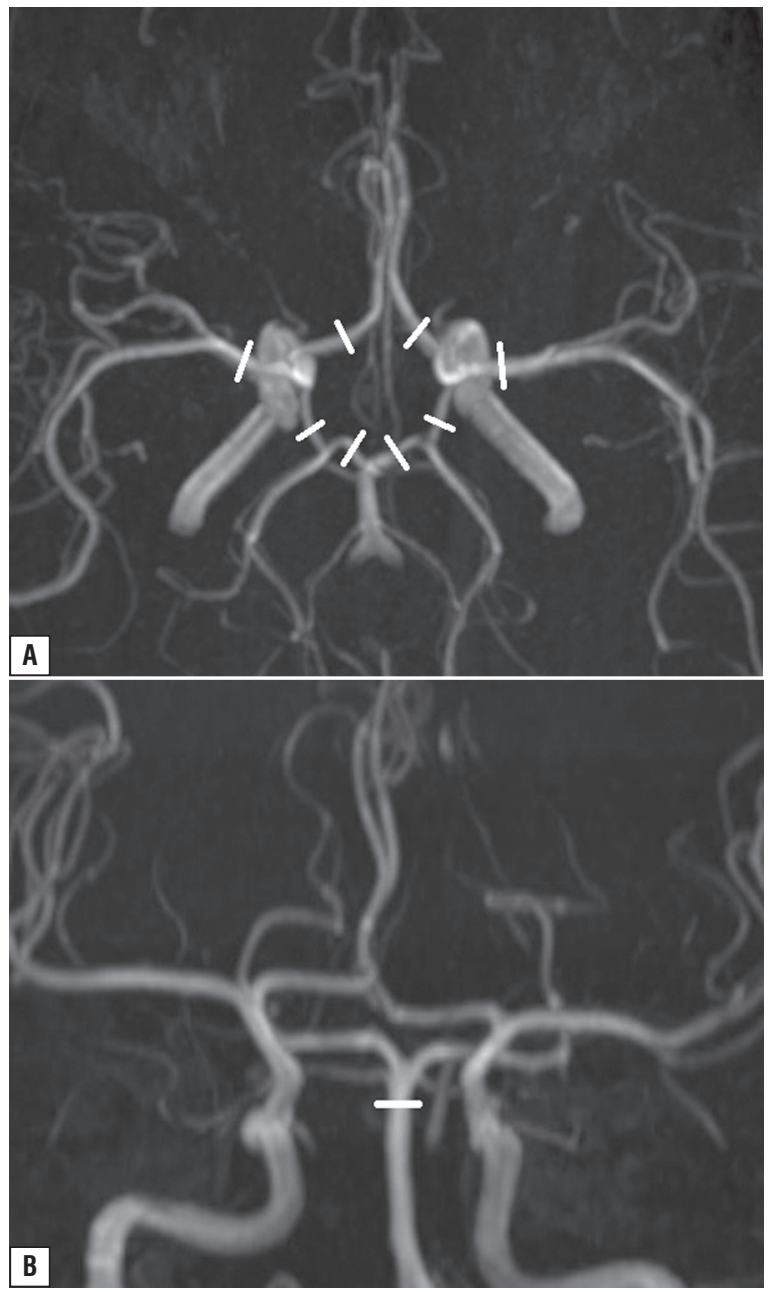

Figure 1. The vascular measurements were made in these regions, the transverse (A) and AP projection (B).

of the vascular measurements and for standardisation, the same window settings were used. Magnification rates were kept similar for each case. All variations in the COW were recorded. The diameters of the critical arteries of the COW were measured. The diameter of the vessels were measured as fallows; in the internal carotid arteries (ICA) in the cavernous segment, in A1 (anterior cerebral artery first segment), in M1 (mid-cerebral artery first segment) and in P1 (posterior cerebral artery first segment) in the first $1 \mathrm{~cm}$ segment, in the basilar arteries in the just inferior of where basilar artery gives branch to superior cerebellar artery, in the posterior communicating artery ( $\mathrm{PCOA}$ ) and in the mid-section between the persistent trigeminal artery (Fig. 1A, B). It is considered as normal when the diameters of $\mathrm{A} 1, \mathrm{M} 1$ and $\mathrm{P} 1$ are larger than $1 \mathrm{~mm}$. 
Table 1. Configuration of the circle of Willis arteries

\begin{tabular}{lc}
\hline & No. (\%) \\
\hline Adult configuration & $326(85 \%)$ \\
Foetal configuration: & $50(13 \%)$ \\
$\quad$ Bilateral & $2(0.5 \%)$ \\
$\quad$ Right & $20(5.2 \%)$ \\
$\quad$ Left & $28(7.3 \%)$ \\
Transitional configuration: & $8(2 \%)$ \\
$\quad$ Right & $4(1 \%)$ \\
\multicolumn{1}{c}{ Left } & $4(1 \%)$ \\
Total & $384(100 \%)$ \\
\hline
\end{tabular}

\section{Statistical analysis}

The statistical analyses of the study data were made with SPSS version 20.0 software (SPSS, Chicago, IL, USA). The demographic findings were recorded. Data were stated as mean \pm standard deviation (SD), number (n) and percentage (\%). The Independent Samples t-test was used for comparison of the group means. A value of $p<0.05$ was accepted as statistically significant.

\section{RESULTS}

In the morphological evaluation, adult configuration of the COW was determined in 326 (85\%) cases and foetal configuration in $50(13 \%)$ cases. Of these, $2(0.5 \%)$ were bilateral, $20(5.2 \%)$ were on the right side and 26 (7.3\%) were on the left side. Transitional configuration was seen in $8(2 \%)$ cases, of which 4 were right-side and 4 were left-side (Table 1).

On the MRA applied to 384 patients, the COW was normal in 328 (85.4\%). In the remaining 56 (14.6\%) cases, agenesis was determined in 62 vessels. These were agenesis in 6 right $A 1,2$ left $A 1,10$ right $P 1$, 6 left P1, 14 right PCoA, 10 left PCoA and 14 bilateral PCoA (Fig. 2A, B). In 2 cases persistent trigeminal artery variation was determined, 1 on the right and 1 on the left (Fig, 3A, B). In 1 case, both A1 were seen to be joined at the level of the anterior communicating artery and continued as a single A2. The data related to the variations are shown in Table 2.

The mean calibrations of the vessels were measured as $2.85 \mathrm{~mm}$ in the basilar artery, $4.24 \mathrm{~mm}$ in the right ICA, $4.32 \mathrm{~mm}$ in the left ICA, $1.58 \mathrm{~mm}$ in the right $A 1,1.64 \mathrm{~mm}$ in the left $A 1,2.13 \mathrm{~mm}$ in the right $M 1,2.10 \mathrm{~mm}$ in the left $M 1,1.80 \mathrm{~mm}$ in the right $P 1$, $1.88 \mathrm{~mm}$ in the left P1, $1.12 \mathrm{~mm}$ in the right PCoA, and $1.12 \mathrm{~mm}$ in the left PCoA. The data related to the vascular diameters are shown in Table 3.

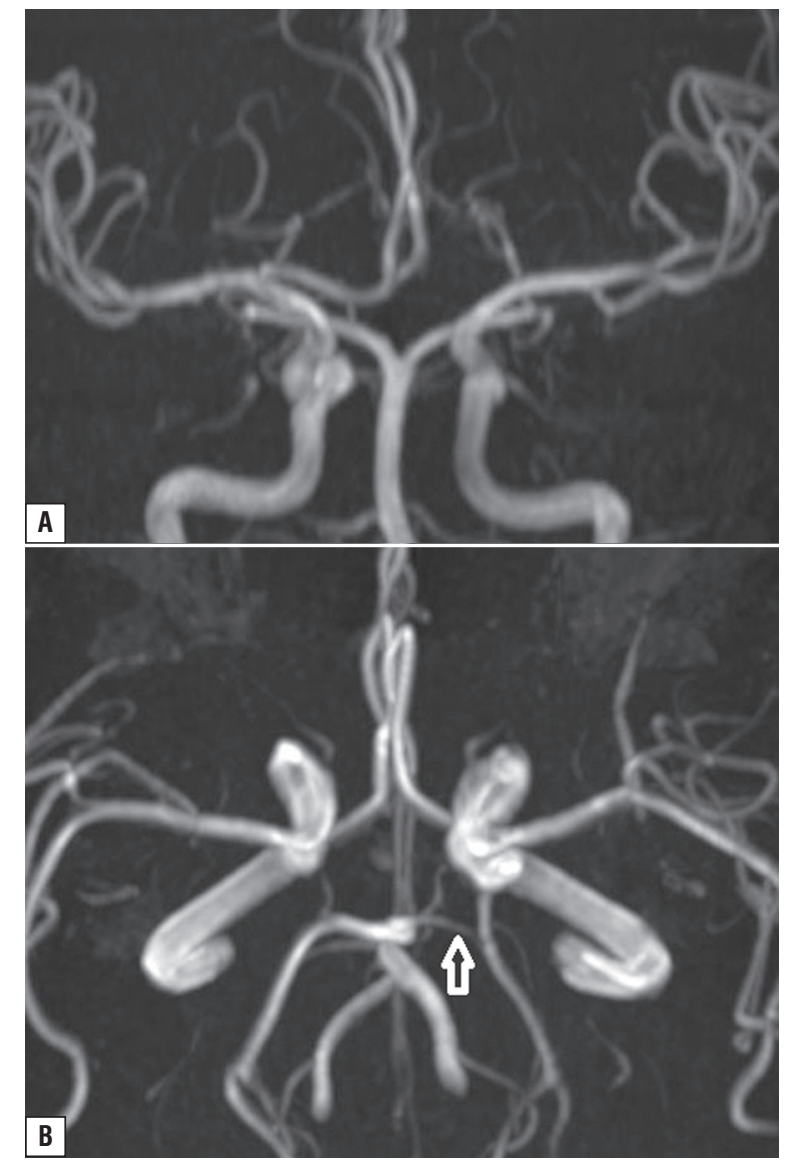

Figure 2. A. Left A1 agenesis on anteroposterior projection magnetic resonance imaging maximal intensity project image, B. Left $\mathrm{P} 1$ agenesis, the arrow shows superior cerebellar artery.

\section{DISCUSSION}

Incomplete COW anomaly was determined at the rate of $14.6 \%$ in the current study, which is significantly lower than the rate of $29 \%$ reported by Karataş et al. [5] from a study applied with CTA. This difference could be related to the lower mean age of the current study population (30 years) compared to that of the Karataş et al. [5] study population. As the mean age of the Karataş et al. study population was 60 years, atherosclerotic occlusion or narrowing of some vessels in the COW which occurs at that age, could have affected the higher rate. Similarly, in a study by El-Barhoun et al. [1] of 171 subjects, 70 (40.9\%) of whom were aged over 60 years, adult configuration of WP was determined in $84.4 \%$ and foetal configuration in $13.6 \%$. These rates were found to be extremely close to the rates of $82 \%$ and $17 \%$ in a study by Karataş et al. [5] applied with CTA. 


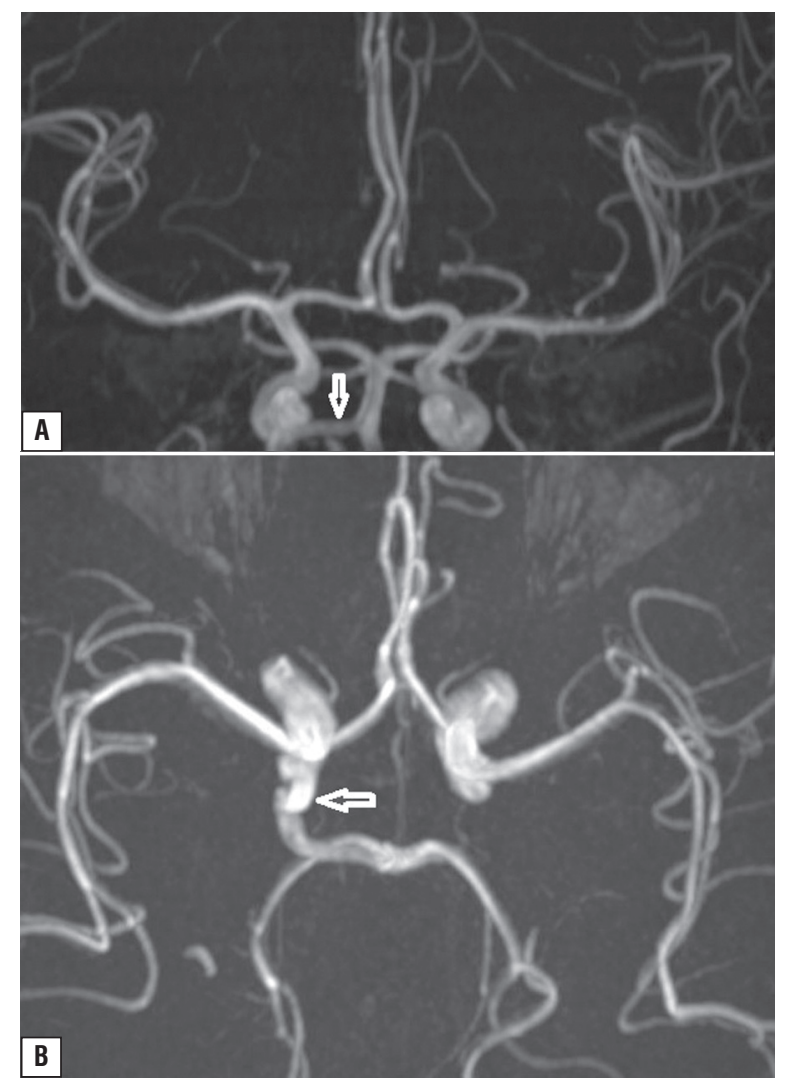

Figure 3. A, B. White arrows indicate right primitive persistent trigeminal artery.

Table 2. Variation $(\mathrm{n}=56)$

\begin{tabular}{lc}
\hline Variation & Number \\
\hline Right A1 agenesis & 6 \\
Left A1 agenesis & 2 \\
Right P1 agenesis & 10 \\
Left P1 agenesis & 6 \\
Right PCoA agenesis & 14 \\
Left PCoA agenesis & 10 \\
Bilateral PCoA agenesis & 14 \\
Right PTA & 1 \\
Left PTA & 1 \\
Total & 64 \\
\hline
\end{tabular}

A1 — first segment of the anterior cerebral artery; P1 — first segment of the posterior cerebral artery; $\mathrm{PCoA}$ - posterior communicating artery; PTA — persistent trigeminal artery

Another finding of the current study was that the posterior circulation of the incomplete structure was significantly high compared to the anterior circula- tion. Of the 62 cases of agenesis, 54 (87\%) were in the posterior circulation. Li et al. [8] determined that agenesis was seen more often in the posterior circulation than in the anterior in a Chinese population. In a study by Krabbe-Hartkamp et al. [7] complete structure was determined in the anterior circulation in $74 \%$, in the posterior circulation in $52 \%$ and in $63 \%$ of all the cases.

Another striking finding of the current study was the distribution of the variations. Of the 62 variations in 56 cases, 38 (59.37\%) were PCoA agenesis, of which 14 were on the right, 10 on the left and 14 bilateral. In a recent study by Gunnal et al. [3], the vessel with the most variation was determined to be the PCoA at $50 \%$.

There is ongoing debate in literature about the threshold diameter value for hypoplasia. KrabbeHartkamp et al. [7] recommended a threshold value of $0.8 \mathrm{~mm}$ for hypoplasia, although there are studies which have recommended a threshold value of $1 \mathrm{~mm}[8,10]$. In the current study, the mean calibration values were measured for right and left sides as M1, 2.10-2.13 mm; P1, 1.79-1.80 mm; A1, 1.58-1.64 mm; PCoA, $1.12 \mathrm{~mm}$. We believe that it is not appropriate to use the same threshold value for hypoplasia in vessels with different calibrations. Although it is not practical to use a different threshold value for each vessel, it would seem to be more plausible to use the percentile values produced from the results obtained from the vessel diameter measurements. The comparisons of the COW artery calibrations in some series are shown in Table 4.

Ghazali et al. [2] tested digital subtraction angiography with 3D TOF MRA in the comparison of the morphology of COW arteries. With the exception of PCOA, MRA was found to be a more sensitive method in the evaluation of the other COW arteries. In this respect, MRA seems to be more advantageous than CTA, as it does not require the use of contrast substance, it does not use ionising radiation, the processing of data is easy and there is not the visualisation of pure bone artefacts as seen on computed tomography in the posterior fossa and particularly in the base of the skull. Motion artefacts constitute the greatest handicap. In the current study, of the total 424 cases, 40 were excluded from the study because of artefacts related to movement. This comprised $9.33 \%$ of all the images. 
Table 3. The reference values measured in the circle of Willis arteries

\begin{tabular}{llllllllll}
\hline & Min & Mean & Max & $\mathbf{5 \%}$ & $\mathbf{2 5 \%}$ & $\mathbf{5 0 \%}$ & $\mathbf{7 5 \%}$ & $\mathbf{9 5 \%}$ \\
\hline BA & 1.50 & 2.85 & 3.70 & 2.30 & 2.70 & 2.80 & 3.00 & 3.30 \\
RICA & 2.50 & 4.24 & 5.60 & 3.20 & 3.80 & 4.20 & 4.70 & 5.20 \\
LICA & 3.00 & 4.32 & 6.10 & 3.40 & 3.80 & 4.20 & 4.80 & 5.30 \\
RA1 & 1.00 & 1.58 & 2.40 & 1.20 & 1.40 & 1.60 & 1.70 & 2.00 \\
LA1 & 1.00 & 1.60 & 2.80 & 1.20 & 1.40 & 1.60 & 1.80 & 2.10 \\
RM1 & 1.50 & 2.13 & 3.20 & 1.70 & 1.90 & 2.10 & 2.30 & 2.60 \\
LM1 & 1.40 & 2.10 & 3.20 & 1.70 & 1.90 & 2.10 & 2.30 & 2.70 \\
RPCoA & 0.70 & 1.12 & 1.90 & 0.70 & 1.00 & 1.10 & 1.30 & 1.50 \\
LPCoA & 0.70 & 1.12 & 1.80 & 0.70 & 1.00 & 1.10 & 1.30 & 1.50 \\
RP1 & 1.10 & 1.80 & 2.60 & 1.40 & 1.70 & 1.80 & 1.90 & 2.30 \\
LP1 & 1.00 & 1.79 & 2.80 & 1.30 & 1.60 & 1.80 & 2.00 & 2.20 \\
\hline
\end{tabular}

BA — basilar artery; RICA — right internal carotid artery; LICA — left internal carotid artery; RA1 — first segment of the right anterior cerebral artery; LA1 — first segment of the left anterior cerebral artery; RM1 — first segment of the right middle cerebral artery; LM1 — first segment of the left middle cerebral artery; RPCoA — right posterior communicating artery; LPCoA - left posterior communicating artery; RP1 — first segment of the right posterior cerebral artery; LP1 — first segment of the left posterior cerebral artery

Table 4. Circle of Willis artery calibration in some studies and our study

\begin{tabular}{lcccccc}
\hline & BA & $\begin{array}{c}\text { ICA } \\
\text { (R-L) }\end{array}$ & $\begin{array}{c}\text { MCA } \\
(\mathbf{R}-\mathbf{L})\end{array}$ & $\begin{array}{c}\text { PCA } \\
\text { (R-L) }\end{array}$ & $\begin{array}{c}\text { ACA } \\
\text { (R-L) }\end{array}$ & $\begin{array}{c}\text { PCoA } \\
\text { (R-L) }\end{array}$ \\
\hline El Barhoun [1] & $3.1-3.3$ & $4.5-4.4$ & $3-2.9$ & $2-2$ & 2.2 & - \\
Karataş [5] & - & - & - & $2.22-2.12$ & $2.15-2.26$ & $1.3-1.27$ \\
Gunnel [3]* & 3 & 4.9 & - & 2.8 & 2.8 & 2.1 \\
Our study & 2.85 & $4.24-4.32$ & $2.13-2.10$ & 1.8 & $1.58-1.60$ & 1.12 \\
\hline
\end{tabular}

${ }^{*}$ This is a cadaveric study; R — right, L — left; ACA — anterior cerebral artery; BA — basilar artery; ICA — internal carotid artery; MCA — middle cerebral artery; PCA — posterior cerebral artery; $\mathrm{PCOA}$ - posterior communicating artery

There were some limitations to the current study. First, as the vessel calibrations became narrower, measurement became more difficult. To overcome this, the measurements were made at similar magnifications. Second is that when the imaging window changed, there were changes in the vessel diameter measurements. Therefore, no change was made to the original window settings in any case. A third limitation was that the images were evaluated by a single observer, so there could be no evaluation of inter and intra-observer differences.

\section{CONCLUSIONS}

Circle of Willis variations may show geographic and ethnic differences. Knowledge of the frequency and types of variation in the population is important for neurosurgeons and in radiological interventional procedures as a guide for entry and in respect of collateral which could develop later.

\section{REFERENCES}

1. El-Barhoun EN, Gledhill SR, Pitman AG. Circle of Willis artery diameters on MR angiography: an Australian reference database. J Med Imaging Radiat Oncol. 2009; 53(3): 248-260, doi: 10.1111/j.1754-9485.2009.02056.x, indexed in Pubmed: 19624291.

2. Ghazali RM, Shuaib IL. Comparison Between 3D TOF Magnetic Resonance Angiography and Intraarterial Digital Subtraction Angiography in Imaging the Circle of Willis. Malays J Med Sci. 2003; 10(1): 37-42, indexed in Pubmed: 23365498.

3. Gunnal SA, Farooqui MS, Wabale RN. Anatomical variations of the circulus arteriosus in cadaveric human brains. Neurol Res Int. 2014; 2014: 687281, doi: 10.1155/2014/687281, indexed in Pubmed: 24891951.

4. Hoksbergen AWJ, Majoie CBL, Hulsmans FJH, et al. Assessment of the collateral function of the circle of Willis: three-dimensional time-of-flight MR angiography compared with transcranial color-coded duplex sonography. AJNR Am J Neuroradiol. 2003; 24(3): 456-462, indexed in Pubmed: 12637297.

5. Karatas A, Coban G, Cinar C, et al. Assessment of the Circle of Willis with Cranial Tomography Angiography. 
Med Sci Monit. 2015; 21: 2647-2652, doi: 10.12659/ MSM.894322, indexed in Pubmed: 26343887.

6. Kominami M, Yamada N, Imakita S, et al. [MR angiography of steno-occlusive lesions of intracranial arteries: a comparative study between turbo MRA and conventional MRA]. Nihon Igaku Hoshasen Gakkai Zasshi. 1999; 59(11): 504-509, indexed in Pubmed: 10536445.

7. Krabbe-Hartkamp MJ, van der Grond J, de Leeuw FE, et al. Circle of Willis: morphologic variation on three-dimensional time-of-flight MR angiograms. Radiology. 1998; 207(1): 103-111, doi: 10.1148/radiology.207.1.9530305, indexed in Pubmed: 9530305.
8. Li Qi, Li J, Lv F, et al. A multidetector CT angiography study of variations in the circle of Willis in a Chinese population. J Clin Neurosci. 2011; 18(3): 379-383, doi: 10.1016/j.jocn.2010.07.137, indexed in Pubmed: 21251838.

9. Macchi C, Catini C, Federico C, et al. Magnetic resonance angiographic evaluation of circulus arteriosus cerebri (circle of Willis): a morphologic study in 100 human healthy subjects. Ital J Anat Embryol. 1996; 101(2): 115-123, indexed in Pubmed: 8997907.

10. Nolte J. The Human Brain. An Introduction to its Functional Anatomy. 5th edn. Mosby, St. Louis 2002. 